\title{
Sophisticated finite strip for vibration analysis of a rotating cylindrical shell
}

\author{
Ivo Senjanović, Ivan Ćatipović, Neven Alujević ${ }^{1}$, Nikola Vladimir, Damjan Čakmak ${ }^{1, *}$ \\ ${ }^{1}$ Fakultet strojarstva i brodogradnje, Sveučilište u Zagrebu, Ivana Lučića 5, 10000 Zagreb, Croatia
}

\begin{abstract}
In this article a two-node finite strip with eight degrees of freedom for free vibration analysis of pre-stressed rotating cylindrical shells is formulated. The circumferential mode shape profiles are described exactly using trigonometric functions. The axial mode shape profiles are approximated by bar and beam shape functions for membrane and bending displacements, respectively. In this way a semi-analytical formulation is facilitated so that the discretization is required only in the axial direction. The developed finite strip is validated by comparisons with analytical results. An excellent agreement is observed both for stationary and rotating shells.
\end{abstract}

\section{Introduction}

The theory of shells and plates has been covered in a systematic manner in a number of books [1-8]. For example, an instructive approach to thin shell theory, written in a relatively simple way and adapted to the engineering level for practical usage, is presented in [9].

The general theory and specific discussions regarding shells of revolution exposed to uniform load can be found in [10]. Such problems are more particular for submarine or aircraft pressure hull designs with pronounced axial symmetry [11-13].

It is often the case that axisymmetric shells rotate around the axis of symmetry [14-27]. For example, rotating shells of revolution are found in engineering practice in rotor systems of gas turbine engines, highspeed centrifugal separators, rotating satellite structures, and automotive tires. Rotation makes their dynamic behaviour significantly more complex. One of the first investigations into vibration of rotating structures has been carried out by Bryan [14]. He studied vibrations of a rotating ring and described the travelling modes phenomenon. These phenomena result from the Coriolis effect, as shown on the example of infinitely long rotating cylindrical shells, $[15,16]$ as well as on finite rotating cylinders $[17,18]$. An experimental study on flexural vibrations of a thin rotating ring is given in [19]. Furthermore, the influence of a pre-stress on free vibrations of rotating cylinders has been studied in [20].

Huang and Soedel [21] used the nonlinear straindisplacement relationships [13], to formulate the corresponding set of differential equations of motion of a rotating cylindrical shell. They solved the free and forced vibration problem of a simply supported cylindrical shell exactly, by assuming simple sine and cosine displacement functions of the circumferential and axial variables. In this case, postulating the eigenvalue - eigenvector problem results in a characteristic polynomial of sixth order. Its solution gives three positive and three negative natural frequencies.

If the shell does not rotate, the polynomial is bicubic. There are three pairs of positive/negative frequencies characterised by the same absolute value. This is physically explained through pairs of backward and forward rotating modes. The two modes of a pair rotate with the same speed in opposite directions and thus superimpose into a stationary mode. The reason why there are three pairs of modes and natural frequencies is that there are three types of dominant modes: bending (radial), longitudinal (axial), and shear (circumferential).

If the shell rotates around its axis with a constant speed, then the polynomial is no longer bi-cubic. The odd polynomial coefficients occur. The positive and negative natural frequencies have distinct absolute values and the so-called frequency veering (bifurcation) occurs. This means that the forward and backward rotating modes no longer rotate with the same rotation speed and thus cannot superimpose into the stationary modes (standing waves). As a result, with spinning shells the modes rotate independently. For example, this phenomenon comes about with tires rolling over the road surface [22-26]. This significantly influences the overall NVH (Noise and Vibration Harshness) characteristics of the vehicle.

It is very common in the literature on vibration of cylindrical shells, either rotating or stationary, to assume that the two ends of the shell are simply supported [21, $27,28]$. This type of boundary support is sometimes referred to as shear diaphragm set of boundary conditions $[8,9]$. The reason why this set of boundary conditions is often used is that it is probably the only one for which a relatively simple solution can be obtained analytically. In other words, mode shapes assumed as

\footnotetext{
* Corresponding author: damjan.cakmak@,fsb.hr
} 
appropriate products of trigonometric functions of the circumferential and axial variables, usually satisfy both the differential equations and the boundary conditions. This results in a mathematically convenient model.

However, such a model is not necessarily suitable to describe a particular engineering problem. For example, although it is very instructive to investigate dynamics of rotating tires assuming simply supported edges of a tire tread-band [21, 27, 28], it is difficult to accept, from an engineering point of view, that the tire sidewall is infinitely stiff in radial or tangential directions $[25,26]$.

In case of cylindrical shells with boundary conditions other than simply supported, the mathematics significantly complicates. Closed form solutions become difficult to obtain. A number of investigations have been undertaken to tackle this problem [29-37]. One of the solutions has been obtained by assuming shell displacement field as a product of Fourier series in the axial direction and trigonometric functions in the circumferential direction [32]. This procedure has been recently extended to rotating cylindrical shells [33]. The problem of free vibration of rotating cylindrical shell, having arbitrary boundary conditions, can be also solved by employing the Rayleigh-Ritz method. Such a solution, using characteristic orthogonal polynomials for displacements variations along the axial direction, can be found in [34].

A completely analytical solution for free vibrations of a rotating cylindrical shell with arbitrary boundary conditions has been recently offered in [35]. The equations of motion in [35] are based on the straindisplacement relationships of Hermann and Armenakas [13]. However, the solution, although exact, requires a rather complicated procedure of the undetermined shell length to perform the analysis.

In this paper, a semi-analytical approach is used which is based on the theory of finite strips [38]. A new, sophisticated finite strip for modelling pre-stressed rotating cylindrical shells is formulated. This methodology benefits from describing the shell displacement field in the circumferential direction exactly so that the discretization is only needed in the axial direction. The energy approach is used with the strain-displacement relationships given in $[13,21]$ to develop and validate such a special finite strip. The strip element is deliberately made quite simple including two nodes and eight degrees of freedom (DOF). Its reliability is checked in a number of numerical examples by comparison of the numerical with analytical results. An excellent agreement is observed for all boundary conditions considered.

\section{The finite strip}

\subsection{Strain and kinetic energies}

A thin cylindrical shell rotating around its axis of symmetry with constant angular speed $\Omega$, is shown schematically in Fig. 1 a). The shell dimensions are the following: $L$ is the length, $a$ is the radius, $h$ is the thickness. The shell mid-surface is defined in the cylindrical coordinate system, where $x$ and $\varphi$ are the axial and angular coordinates, respectively. The displacement of a point $P$ on the mid-surface, whose position is defined by $x$ and $\varphi$, is specified by axial, tangential and radial displacement components, $u, v$ and $w$, respectively.
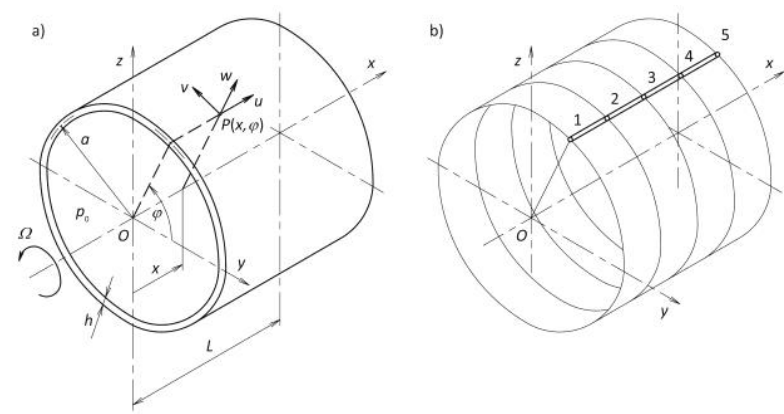

Fig. 1. Rotating cylindrical shell with: a) the main dimensions and the displacement components. b) finite strips with nodes

The strain energy stored in an infinitesimal shell element of thickness $h$ is

$$
\mathrm{d} E_{s}=F_{s}(x, \phi, t) \mathrm{d} x \mathrm{~d} \phi
$$

where $F_{s}$ is the strain energy density function. The kinetic energy stored in an infinitesimal shell element is given by

$$
d E_{k}=F_{k}(x, \phi, t) \mathrm{d} x \mathrm{~d} \phi,
$$

where the kinetic energy density function $F_{k}$ is a product of the specific mass per unit area and the total velocity squared. The total velocity consists of the velocity components in the axial, circumferential and radial directions. It also includes contributions due to the shell rotation.

The total potential and kinetic energy of a general cylindrical shell is given by

$$
E=\int_{0}^{2 \pi} \int_{0}^{L}\left[F_{s}(x, \phi, t)+F_{k}(x, \phi, t)\right] \mathrm{d} x \mathrm{~d} \phi,
$$

where the energy density functions $F_{s}$ and $F_{k}$ are denoted in Eq. (1) and (2), respectively.

For a cylindrical shell with a closed cross-section and arbitrary boundary conditions at the two ends (at $x=0$ and $x=L$, where $L$ is the length of the shell), the three displacement components can be assumed in the form

$$
\begin{aligned}
& u(x, \phi, t)=U(x) \cos (n \phi+\omega t), \\
& v(x, \phi, t)=V(x) \sin (n \phi+\omega t), \\
& w(x, \phi, t)=W(x) \cos (n \phi+\omega t),
\end{aligned}
$$

where $\omega$ is a natural frequency.

\subsection{Stiffness matrix}

In this section the properties of the finite strip of a rotating cylindrical shell are developed by employing the energy approach. The rotating cylindrical shell is modelled by a number of finite strips, as shown in Fig. $1 \mathrm{~b})$. Due to the trigonometric variation of the displacement field in the circumferential direction, the dynamic behaviour of the finite strip becomes representative by its axial generatrix which behaves like a sophisticated beam. The stiffness matrix is obtained using the element strain energy from Eq. (1) 


$$
E_{s}=\int_{0}^{2 \pi} \int_{0}^{l} F_{s}(x, \phi, t) \mathrm{d} x \mathrm{~d} \phi,
$$

where the expression for $F_{s}$ can be found in [39], and $l$ is the strip width. Substituting the assumed displacement functions (4) into the expression for $F_{s}$ and integrating Eq. (5) over the circumference $\phi$, the strain energy is obtained in the form

$$
\begin{aligned}
E_{s}=\int_{0}^{l} & {\left[\begin{array}{l}
\frac{1}{2} a_{1}\left(U^{\prime}\right)^{2}+\frac{1}{2} a_{2} U^{2}+\frac{1}{2} a_{3}\left(V^{\prime}\right)^{2}+ \\
\frac{1}{2} a_{4} V^{2}+a_{5} U^{\prime} V+a_{6} U V^{\prime}+ \\
+ \\
+\frac{1}{2} b_{1}\left(W^{\prime \prime}\right)^{2}+\frac{1}{2} b_{2}\left(W^{\prime}\right)^{2}+\frac{1}{2} b_{3} W^{2}+ \\
+b_{4} W^{\prime \prime} W+b_{5} W U^{\prime}+b_{6} W^{\prime} V^{\prime}+b_{7} W V+b_{8} W^{\prime \prime} V
\end{array}\right] \mathrm{d} x, }
\end{aligned}
$$

where the coefficients $a_{1} \ldots a_{6}$ and $b_{1} \ldots b_{8}$ can be found in [39]. The strain energy (6) is time-invariant. This is due to the fact that the rotating modes are characterised by fixed profiles which rotate along the circumference.

A relatively simple finite strip with two-nodal lines is introduced next. The amplitudes of the displacement components, Eqs. (4), are approximated by the following interpolation functions:

$$
\begin{aligned}
& U(x)=\sum_{j=1}^{2} U_{j} g_{j}(x), \\
& V(x)=\sum_{j=1}^{2} V_{j} g_{j}(x), \\
& W(x)=\sum_{j=1}^{4} \Delta_{j} f_{j}(x),
\end{aligned}
$$

where

$$
\{U\}=\left\{\begin{array}{c}
U_{1} \\
U_{2}
\end{array}\right\}, \quad\{V\}=\left\{\begin{array}{c}
V_{1} \\
V_{2}
\end{array}\right\}, \quad\{\Delta\}=\left\{\begin{array}{c}
W_{1} \\
\Theta_{1} \\
W_{2} \\
\Theta_{2}
\end{array}\right\},
$$

are vectors of the amplitudes of the nodal line displacements, i.e. nodal displacements for short, Fig. 2.

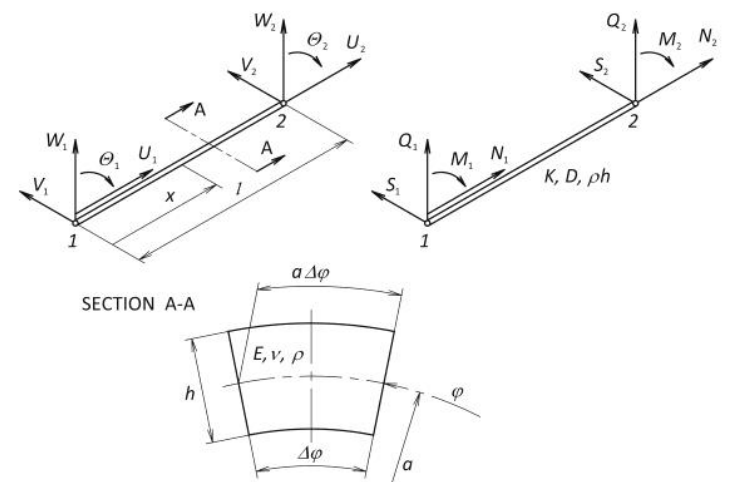

Fig. 2. Nodal displacements and nodal forces of the finite strip and its cross-section.

For membrane displacements, ordinary polynomial bar shape functions are used. For bending displacements, beam shape functions (Hermitian polynomials) are used:

$$
\begin{aligned}
& g_{1}=1-\xi, \quad g_{2}=\xi \\
& f_{1}=1-\xi^{2}(3-2 \xi), \quad f_{2}=l \xi(1-\xi)^{2}, \\
& f_{3}=\xi^{2}(3-2 \xi), \quad f_{4}=-l \xi^{2}(1-\xi),
\end{aligned}
$$

where $\xi=x / l$ is the dimensionless axial coordinate.
Substituting expressions (7) into (6) and differentiating the strain energy with respect to the nodal displacements, a system of eight algebraic equations is obtained, which can be written in the matrix notation:

$$
\frac{\partial E_{s}}{\partial\{\delta\}}=[K]\{\delta\}
$$

where

$$
\langle\delta\rangle=\{\delta\}^{T}=\left\langle\begin{array}{llllllll}
U_{1} & U_{2} & V_{1} & V_{2} & W_{1} & \Theta_{1} & W_{2} & \Theta_{2}
\end{array}\right\rangle
$$

is the vector of the nodal displacements, Fig. 2, and $[K]$ is the strip stiffness matrix. The stiffness matrix consists of eleven submatrices as follows:

$$
[K]=\left[\begin{array}{ccc}
a_{1}[K]_{1}+a_{2}[K]_{2} & a_{5}[K]_{3}+a_{6}[K]_{3}^{T} & b_{5}[K]_{8} \\
a_{5}[K]_{3}^{T}+a_{6}[K]_{3} & a_{3}[K]_{1}+a_{4}[K]_{2} & b_{6}[K]_{9}+b_{7}[K]_{10}+b_{8}[K]_{11} \\
b_{5}[K]_{8}^{T} & b_{6}[K]_{9}^{T}+b_{7}[K]_{10}^{T}+b_{8}[K]_{11}^{T} & b_{1}[K]_{4}+b_{2}[K]_{5}+b_{3}[K]_{6}+b_{4}[K]_{7}
\end{array}\right]
$$

Submatrices $[K]_{i}, i=1,2 \ldots 11$ represent integrals of a different combination of the shape function products. They are calculated in the closed form and given in [39] with some comments on their physical meaning. The stiffness matrix in fact incorporates a geometric stiffness matrix, which is due to the initial tension forces, and which tend to increase the natural frequencies [39].

\subsection{Mass matrices}

Mass matrices are determined from the shell kinetic energy from Eq. (2)

$$
E_{k}=\int_{0}^{2 \pi} \int_{0}^{l} F_{k}(x, \phi, t) \mathrm{d} x \mathrm{~d} \phi .
$$

The kinetic energy density function $F_{k}$ is given by

$$
F_{k}(x, \phi, t)=\frac{1}{2} a \rho h\left\{\left(\frac{\partial u}{\partial t}\right)^{2}+\left[\frac{\partial v}{\partial t}+(a+w) \Omega\right]^{2}+\left(\frac{\partial w}{\partial t}-v \Omega\right)^{2}\right\}
$$

Substituting (14) and (4) into (13), one obtains after integration over the circumference angle

$$
E_{k}=\pi a \rho h \int_{0}^{l}\left[\begin{array}{l}
\frac{1}{2} \omega^{2} U^{2}+\frac{1}{2}\left(\omega^{2}+\Omega^{2}\right) V^{2}+ \\
+\frac{1}{2}\left(\omega^{2}+\Omega^{2}\right) W^{2}+2 \omega \Omega W V+a^{2} \Omega^{2}
\end{array}\right] \mathrm{d} x
$$

The kinetic energy (15) is also time invariant for the same reason of rotating fixed axial mode profiles.

Furthermore, substituting expressions (7) into (15) and differentiating the kinetic energy with respect to the nodal displacements yields

$$
\begin{aligned}
& \left\{\frac{\partial E_{k}}{\partial U_{i}}\right\}_{i=1,2}=\pi a \rho h \omega^{2}[K]_{2}\{U\}, \\
& \left\{\frac{\partial E_{k}}{\partial V_{i}}\right\}_{i=1,2}=\pi a \rho h\left(\omega^{2}+\Omega^{2}\right)[K]_{2}\{V\}+2 \pi a \rho h \Omega \omega[K]_{10}\{\delta\}, \\
& \left\{\frac{\partial E_{k}}{\partial \Delta_{i}}\right\}_{i=1,2,3,4}=\pi a \rho h\left(\omega^{2}+\Omega^{2}\right)[K]_{6}\{\delta\}+2 \pi a \rho h \Omega \omega[K]_{10}^{T}\{V\} .
\end{aligned}
$$

Submatrices $[K]_{i}, i=2,6,10$, have been calculated and can be found in [39]. The system of algebraic equations (16) can be written in the matrix form

$$
\frac{\partial E_{k}}{\partial\{\delta\}}=[A]\{\delta\},
$$


where $\{\delta\}$ is a vector of nodal displacements, Eq. (11). Matrix $[A]$ can be separated into three matrices with respect to the power of frequency $\omega$

$$
[A]=[B]+\omega[C]+\omega^{2}[M]
$$

where matrices $[B],[C]$ and $[M]$ are given in [39].

\subsection{The finite strip equation}

By employing the minimum total energy principle, one obtains the finite strip equation in the following form

$$
\{F\}=\left([K]-[B]-\omega[C]-\omega^{2}[M]\right)\{\delta\} .
$$

Matrix $[B]$, which depends on $\Omega^{2}$, reduces the element stiffness due to the rotating mass, whereas the geometric stiffness matrix contained in $[K]$ has the opposite effect. However, the net effect of terms depending on $\Omega^{2}$ is an increase of natural frequencies. Details on how to re-arrange the rows and columns of all matrices in (19) in order to facilitate a chain-like assembly of the strip equations are given in [39].

\section{Numerical examples}

\subsection{Simply supported shell}

In order to illustrate the application of the developed finite strip and to evaluate its accuracy, a number of numerical examples considering various boundary conditions are presented. First, a free vibration analysis a of simply supported cylindrical shell with the following geometric and material properties of rubber is carried out using the example from [35]: $L=0.2 \mathrm{~m}, a=0.1 \mathrm{~m}$, $h=0.002 \mathrm{~m}, E=0.45 \mathrm{GPa}, v=0.45, \rho=1452 \mathrm{~kg} / \mathrm{m}^{3}$. The developed finite strip is validated against an analytical solution [35]. The bifurcation of natural frequencies due to the angular velocity $\Omega$, for a few $m \times n$ natural modes, has been calculated using 20 strip elements, and shown in Fig. 3 for two example modes.
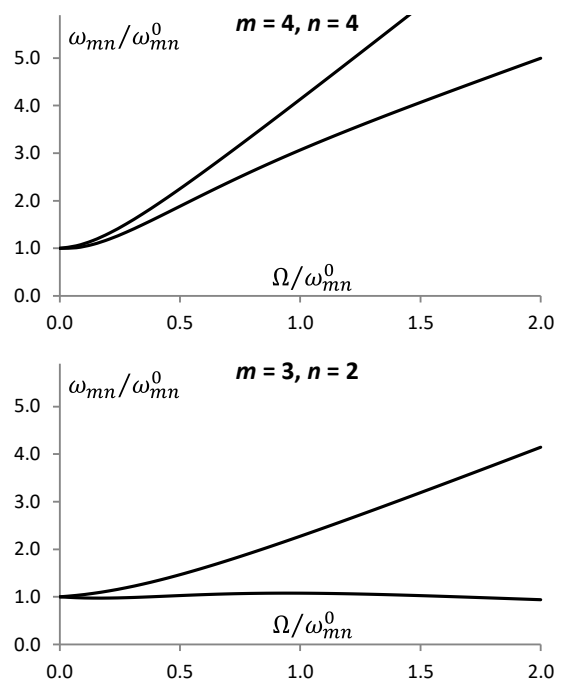

Fig. 3. Bifurcation of normalized natural frequencies of a rotating simply supported cylindrical shell.
Parameter $m$ denotes the number of vibration nodes of the axial mode profile and the parameter $n$ denotes the number of the nodes of the circumferential mode profile. The same results are obtained analytically so that the curves perfectly overlap.

Parametric analysis considering the internal pressure $p_{0}$ is performed next. Fig. 4 shows the increase of natural frequencies due to increased internal pressure. Also here the curves obtained analytically perfectly overlap with those calculated using the present finite strip method.

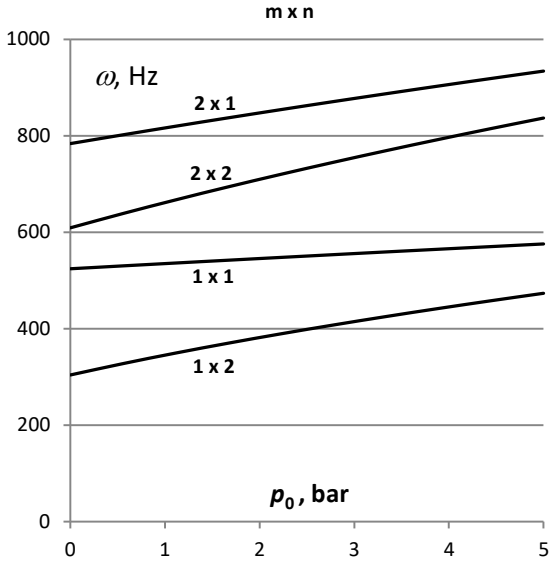

Fig. 4. Increase of natural frequencies of a simply supported cylindrical shell due to internal pressure.

\subsection{Free-Free shell}

Free vibration analysis of the cylindrical shell having the same geometric and material properties as in the previous example is now carried out for the case of free boundary conditions at the two ends of the shell. Two natural modes are shown in Fig. 5.
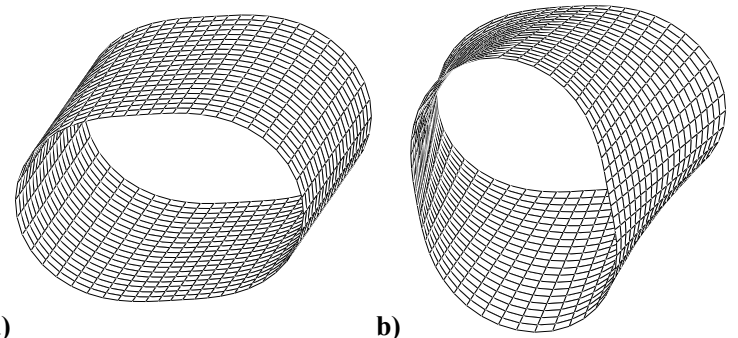

Fig. 5. Natural modes of the free cylindrical shell $(m, n)$ : a) $0 \times 2$ Rayleigh mode, $15.2 \mathrm{~Hz}, \mathrm{~b}) 1 \times 2$ Love mode, $20.7 \mathrm{~Hz}$.

The axial profiles of some characteristic natural modes are also shown in Fig. 6. Natural modes with $m=0$ and $m=1$ are known as Rayleigh-type and Lovetype mode respectively, following the nomenclature suggested in [8]. It is interesting that both symmetric and antisymmetric axial mode profiles are curved at the ends.

The curvature is increased by increasing the wave number $n$ in circumferential direction [35]. This phenomenon manifests the effect of the boundary conditions. A more detailed analysis of the mode shapes of free-free shells can be found in [31], [35-37]. 


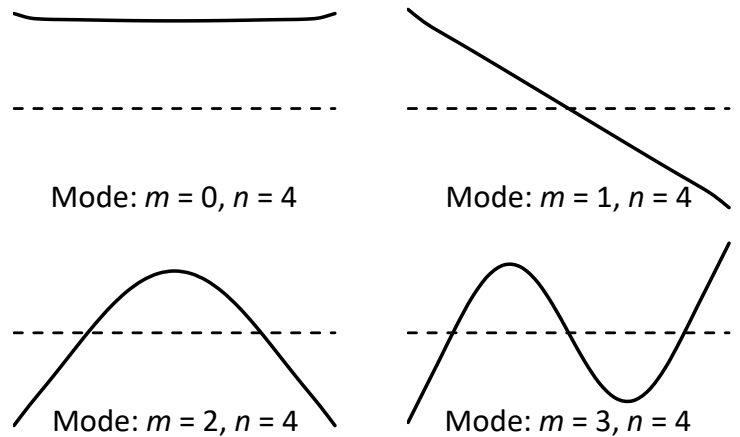

Fig. 6. Natural modes of the free cylindrical shell: axial mode profiles, $\Omega=0, p_{0}=0$

\section{Conclusions}

In this paper, a new two-node finite strip with eight degrees of freedom for free vibration analysis of rotating and pre-stressed cylindrical shells is formulated. In order to develop the stiffness and mass matrices of the finite strip, simple bar and beam shape functions are used. The element stiffness matrix is a sum of an ordinary stiffness matrix and a geometric stiffness matrix. The geometric stiffness matrix is due to the initial tension forces. On the other hand, the inertia matrix is constituted of three mass matrices. The first one is the ordinary mass matrix related to squared natural frequencies (inertia load), the second one is related to squared angular velocity (centrifugal load), and the third mass matrix is related to the multiple of a natural frequency and angular velocity (Coriolis load). The stiffness and mass matrices are constituted of eleven submatrices, which are effectively spatial integrals of products of different combinations of the shape functions and their derivatives. These integrals have been calculated exactly such that the proposed finite strip method can be used without further numerical burden.

The application of the developed finite strip is illustrated by numerical examples considering simply supported and free-free boundary conditions at the two ends of the shell. The reliability of the finite strip is validated by a comparison with a benchmark analytical solution and an excellent agreement is observed. Also, a parametric analysis is performed in order to appreciate the effects of the angular velocity and pre-stress on natural frequencies. It is shown that veering of natural frequencies due to Coriolis effect, as well as their upward shift due to the pressurisation and the centrifugal effect, can be very accurately simulated.

Nowadays, vibrations of rotating tires are extensively investigated primarily due to their importance regarding the Noise and Vibration Harshness (NVH) of a road vehicle. The cylindrical finite strip developed in this paper can be used to model the belt of a rotating tyre. In order to model a tyre sidewall, a conical of toroidal finite strip would be necessary. In order to reduce the number of the finite strips necessary to accurately model a shell structure, a sophisticated finite strip with increased number of nodes and possibly faster convergence may also be considered.
This project has received funding from the European Union's Horizon 2020 research and innovation programme under the Marie Sklodowska-Curie grant agreement no. 657539. This investigation also received funding within the international collaborative project Global Core Research Center for Ships and Offshore Plants (GCRC SOP), established by South Korean Government (MSIP) through the National Research Foundation of South Korea (NRF).

\section{References}

1. S. Timoshenko, S. Woinowsky-Krieger, Theory of Plates and Shells (McGraw-Hill Book Company, New York, 1959)

2. V.V. Novozhilov, The Theory of Thin Elastic Shells (P. Noordhoff, Gromingen, The Netherlands, 1964)

3. W. Flügge, "Statik and Dynamik der Schalen" (Statics and dynamics of shells) (Springer-Verlag, Berlin, 1934)

4. A.L. Goldenveizer, Theory of Thin Shells (Pergamon Press, Elmsford, New York, 1961)

5. V.Z. Vlasov, General Theory of Shells and Its Applications in Engineering (translation from Russian) (NASA TTF-99, U.S. Governmental Printing Office, Washington, D.C., 1964)

6. J.E. Gibson, Linear Elastic Theory of Thin Shells (Pergamon Press, London, 1965)

7. L.H. Donnell, Beams, Plates, and Shells (McGrawHill, New York, 1976)

8. A.W. Leissa, Vibrations of Shells (NASA SP-288, U.S. Government Printing Office, Washington, D.C., 1973)

9. W. Soedel, Vibrations of Shells and Plates, 3rd edition, revised and expanded (Marcel Dekker Inc., New York, 2004)

10. I. Senjanović, Theory of Shells of Revolution (Ship Research Institute, Zagreb, 1972)

11. C.T.F. Ross, Pressure Vessels Under External Pressure: Statics and Dynamics (Elsevier Applied Science, London, 1990)

12. Group of Authors, in E.E. Allmenidinger, (Ed.), Submersible Vehicle System Design (SNAME, Jersey City, 1990)

13. G. Herrmann. A.E. Armenakas, Dynamic Behavior of Cylindrical Shells under Initial Stress, Proc. 4th U.S. Nat. Congr. Appl. Mech, ASME, 203-213, (1962)

14. G.H. Bryan, On the beats in the vibrations of a revolving cylinder or bell. In: Proc. of the Camb. Philos. Soc., 101-111 (1880)

15. R.A. Di Taranto, M. Lessen, Coriolis Acceleration Effect on the Vibration of a Rotating Thin-Walled Circular Cylinder, J. Appl. Mech. 31, 700-701 (1964)

16. A.V. Srinivasan, G.F. Lauterbach, Traveling Waves in Rotating Cylindrical Shells, J. Eng. Ind. ASME 93, 1229-1232 (1971) 
17. A. Zohar, J. Aboudi, The free vibrations of a thin circular finite rotating cylinder, Int. J. Mech. Sci. 15, 269-278 (1973)

18. T. Saito, M. Endo, Vibration of finite length, rotating cylindrical shells, J. Sound Vib. 107, 17-28 (1986)

19. M. Endo, K. Hatamura, M. Sakata, O. Taniguchi, Flexural vibration of a thin rotating ring, J. Sound Vib. 92, 261-272 (1984)

20. J. Padovan, Natural frequencies of rotating prestressed cylinders. J. Sound Vib, 31, 469-482 (1973)

21. S.C. Huang, W. Soedel, On the forced vibration of simply supported rotating cylindrical shells, J. Acoust. Soc. Am. 84(1), 275-285 (1988)

22. C. Gonzalez Diaz, P. Kindt, J. Middelberg, S. Vercammen, C. Thiry, R. Close, J. Leyssens, Dynamic behaviour of a rolling tyre: Experimental and numerical analyses, J. Sound Vib. 364, 147-164 (2016)

23. J. Lee, S. Wang, P. Kindt, B. Pluymers, W. Desmet, Identification of the direction and value of the wave length of each mode for a rotating tire using the phase difference method, Mech. Sys. and Signal Process. 68-69, 292-301 (2016)

24. P. Kindt, C. G. Diaz, S. Vercammen, C. Thiry, J. Middelberg, B. Kimble, J. Leyssens, Effects of rotation on the tire dynamic behavior: Experimental and numerical analyses, Tire Sci. and Tec. 41(4), 248-261 (2013)

25. W.R. Graham, Modelling the vibration of tyre sidewalls, J. Sound Vib. 332(21), 5345-5374 (2013)

26. C. Lecomte, W.R. Graham, M. Dale, A shell model for tyre belt vibrations, J. Sound Vib. 329(10), 1717-1742 (2010)

27. Y.-J. Kim, JS. Bolton, Effects of rotation on the dynamics of a circular cylindrical shell with application to tire vibration. J. Sound Vib. 275, 605621 (2004)

28. L.R, Molisani, R.A. Burdisso, D. Tsihlas, A coupled tire structure/acoustic cavity model. Int. J. Solids Struct. 40, 5125-5138 (2003)

29. K. Forsberg, Influence of boundary conditions on the modal characteristics of thin cylindrical shells, AIAA J. 2(12), 2150-2157 (1964)

30. V.I. Weingarten, On the free vibration of thin cylindrical shells (Aerospace corporation, Systems research and planning division, Report No. TDR.169(3560.30)TN-3, El Segundo, California, 1962)

31. G.B. Warburton, Vibration of thin cylindrical shells, J. Mech. Eng. Sci. 7(4), 399-407 (1965)

32. H. Chung, Free vibration analysis of circular cylindrical shells, J. Sound Vib. 74, 331-350 (1981)

33. S. Sun, S. Chu, D. Cao, Vibration characteristics of thin rotating cylindrical shells with various boundary conditions, J. Sound Vib. 331, 4170-4186 (2012)

34. S. Sun, D. Cao, Q. Han, Vibration studies of rotating cylindrical shells with arbitrary edges using characteristic orthogonal polynomials in the Rayleigh-Ritz method, Int. J. of Mec. Sci. 68, 180189 (2013)

35. N. Alujević, N. Campillo-Davo, P. Kindt, W. Desmet, B. Pluymers, S. Vercammen, Analytical solution for free vibrations of rotating cylindrical shells having free boundary conditions. Eng. Str. 132, 152-171 (2017)

36. N. Alujević, N. Campillo-Davo, P. Kindt, W. Desmet, B. Pluymers, S. Vercammen, A simplified tire model based on a rotating shell, Proceedings of the 4th International Tyre Colloquium, University of Surrey, Surrey (2015)

37. N. Alujević, N. Campillo-Davo, P. Kindt, W. Desmet, B. Pluymers, S. Vercammen, A simplified model of a rotating tire using cylindrical shells with free ends supported by an elastic foundation, Proceedings of ISMA2014, Katholieke Universiteit Leuven, Leuven (2014)

38. Y.K. Cheung, Finite Strip Method in Structural Analysis (Pergamon Press, Oxford, 1976)

39. I. Senjanović, I. Ćatipović, N. Alujević, N. Vladimir, D. Čakmak, A finite strip for the vibration analysis of rotating cylindrical shells, Thin-Wall. Struct. (accepted). 